\title{
KRAS and NRAS Testing in Metastatic Colorectal Cancer in Central Iran (Tehran): A Review on Literature of the Middle East
}

\begin{abstract}
Context: The incidence of colorectal cancer (CRC) in the past three decades in Iran has made it as a major public health burden. Aims: The aim of this study is to report the prevalence of KRAS and NRAS mutations in Iran and the correlation between KRAS mutation status with clinicopathological factors and survival. Materials and Methods: In a cross-sectional study, 144 patients were entered into the study based on the criteria. Age, sex, tumor site, grade, metastasis location, familial history, KRAS/NRAS status, and survival were checked for all patients, and the patients were followed for 1 year. DNA was extracted with FFPE QIAGEN kit and then polymerase chain reaction for amplification of gene segments of KRAS and NRAS genes. Results: The mean age at diagnosis was 52.9 years (range: $27-72$ years) that $39.6 \%$ patients had age $<50$ years and $54.2 \%$ were men. KRAS mutation was significantly more in the patients with age $\geq 50$ compared with KRAS wild type. Furthermore, the 6-month overall survival rate in KRAS mutation patients was significantly more than KRAS wild-type patients. Liver metastasis $(72.9 \%)$ had the highest prevalence of metastasis in the patients, and Grade II with $64.6 \%$ had the most prevalence. Conclusions: The metastatic CRC was more prevalent in men than women, and the mean age varied around 50-60 years. The results showed that the present study had the highest prevalence of KRAS mutation in the Middle East and Pakistan with the lowest prevalence in CRC patients.
\end{abstract}

Keywords: Colorectal cancer, Iran, KRAS, NRAS

\section{Introduction}

Colorectal cancer (CRC) is one of the most common cancers and is the second leading cause of cancer death in men and women in the United States and also is third common cancer in women and fifth in Iranian men. ${ }^{[1]}$ The increasing incidence of CRC in the past three decades in Iran has made it as a major public health burden. ${ }^{[2]}$ In CRC patients, metastases are the main cause of cancer-related mortality. ${ }^{[3]}$ The most common sites of metastasis from colon cancer are the regional lymph nodes, the liver, the lung, and the peritoneum. ${ }^{[3,4]}$ Distant metastatic disease is present in approximately $25 \%$ of patients at initial diagnosis, and half of CRC patients will develop metastatic disease. ${ }^{[5]}$ Most patients with metastatic CRC (mCRC) have an incurable disease. ${ }^{[3]}$ The treatment of $\mathrm{mCRC}$ is one of the biggest successes in recent decades. ${ }^{[6]}$ Targeted cancer therapy is becoming a powerful strategy for the treatment of patients selected on the basis of their molecular characteristics. This is particularly true for patients with

This is an open access article distributed under the terms of the Creative Commons Attribution-NonCommercial-ShareAlike 3.0 License, which allows others to remix, tweak, and build upon the work non-commercially, as long as the author is credited and the new creations are licensed under the identical terms.

For reprints contact: reprints@medknow.com
mCRC ${ }^{[2]}$ RAS mutations are useful markers for predicting responses to anti-epidermal growth factor receptor monoclonal antibodies in mCRC. ${ }^{[2,7]}$ KRAS mutation varies between $20 \%$ and $50 \%$ in the most countries in the world ${ }^{[8]}$ but NRAS mutations are rare and occur in 3\% and $5 \%$ of CRC. ${ }^{[9]}$ The frequency of NRAS mutations and their relationship to clinical, pathologic, and molecular features remains uncertain. ${ }^{[9]}$

The aim of this study is another report from the prevalence of KRAS and NRAS mutations in Iran and the correlation between KRAS mutation status with clinicopathological factors and survival.

\section{Materials and Methods}

\section{Patients}

The cross-sectional study was done in CRC patients in Rasool Akram Hospital, Tehran, in 1 year (April 2015 to April 2016) that 144 patients were entered into the study based on inclusion criteria.

How to cite this article: Shahriari-Ahmadi $A$, Ansarinejad N, Fardad F, Abbaszadeh M, Sadeghi M. KRAS and NRAS testing in metastatic colorectal cancer in Central Iran (Tehran): A review on literature of the middle east. Indian J Med Paediatr Oncol 2018;39:210-4.
Ali

Shahriari-Ahmadi, Nafise Ansarinejad, Farshid Fardad, Mehrdad Abbaszadeh, Masoud Sadeghi ${ }^{1}$

Department of Hematology and Medical Oncology, Rasool Akram Hospital, Iran University of Medical Sciences, Tehran, ${ }^{1}$ Medical Biology Research Center, Kermanshah University of Medical Sciences, Kermanshah, Iran

Address for correspondence: Dr. Masoud Sadeghi, Medical Biology Research Center, Kermanshah University of Medical Sciences, Kermanshah, Iran. E-mail:sadeghi_mbrc@yahoo. com

Access this article online

Website: www.ijmpo.org

DOI: 10.4103/ijmpo.ijmpo_133_17 Quick Response Code:

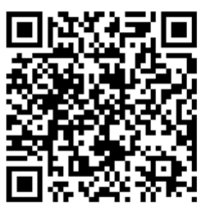




\section{Inclusion criteria}

Patients having $\mathrm{mCRC}$ and history of treatment with chemotherapy regimens were included in the study. Age, sex, tumor site, grade, metastasis location, familial history, KRAS/NRAS status, and survival were checked for all patients. The patients were followed up for 1 year. The overall survival was defined as the length of time from either the date of diagnosis or the start of treatment for cancer until death for any cause or the date of the last follow-up.

\section{Extraction and amplification of KRAS/NRAS}

Mutations and oncogenes of KRAS and NRAS of codons 12 and 13 were checked on fresh frozen and formalin-fixed paraffin-embedded (FFPE) tissues in Partolab Laboratory, Tehran, Iran. After 4-5 cutting with 2-5 $\mu \mathrm{m}$ thickness, DNA was extracted with FFPE QIAGEN kit, and then, polymerase chain reaction (PCR) for amplification of gene segments was done with an initial denaturation at $95^{\circ} \mathrm{C}$ for $11 \mathrm{~min}$, denaturation at $95^{\circ} \mathrm{C}$ for $30 \mathrm{~s}$, fusion at $55^{\circ} \mathrm{C}$ for 30 $\mathrm{s}$, elongation at $72^{\circ} \mathrm{C}$ for $30 \mathrm{~s}$, and end elongation at $72^{\circ} \mathrm{C}$ for 5 min. After electrophoresis of PCR products, a band in the situation of $120 \mathrm{bp}$ was seen that is indicating amplification of gene segment during PCR. To ensure amplified fragment length to confirm the target gene, evaluation was performed by Gene Runner program, and the incision with enzyme was confirmed by MapViewer program. After that, using RFLP technic and suitable enzymes (Bgl1), the status of mutation and wild type was determined. The results again were checked with high-resolution melting analysis. KRAS and NRAS genes were analyzed and sequenced (pyrosequencing) by allele specific.

\section{Statistical analysis}

The analysis was done with IBM SPSS software version 22 (IBM Corp., Armonk, NY, USA) that $t$-test was used for the comparison of means between groups and Chi-square test for other variables. The overall survival was plotted and analyzed by Kaplan-Meier.

\section{Results}

The mean age \pm standard deviation at diagnosis was $52.9 \pm 12.7$ years (range: $27-72$ years) that $39.6 \%$ patients had age $<50$ years and $54.2 \%$ were males [Table 1]. Sigmoid (33.4\%), ascending colon (31.2\%), rectum (20.8\%), descending colon (8.4\%), and transverse colon $(6.2 \%)$ were the highest tumor site in the patients. Grades I (well differentiated), II (moderate differentiated), and III (poorly differentiated) were 16.7\%, 64.6\%, and $18.7 \%$, respectively. In all patients, liver metastasis $(72.9 \%)$ had the highest prevalence, followed by nonregional lymph node and lung (each 10.4\%) and other metastases (6.3\%). Of 144 patients, 15 patients had a familial history of cancer, $72(52.1 \%)$ had KRAS mutation, and $6(4.2 \%)$ had NRAS mutation. In addition, during 1-year follow-up, 51 (35.4\%) patients died for any cause.

\begin{tabular}{|c|c|}
\hline \multicolumn{2}{|c|}{ Table 1: Characteristics of all patients $(n=144)$} \\
\hline Variables & $n(\%)$ \\
\hline \multicolumn{2}{|l|}{ Age (years) } \\
\hline Mean \pm SD & $52.9 \pm 12.7$ \\
\hline Range & $27-72$ \\
\hline$<50$ & $57(39.6)$ \\
\hline \multicolumn{2}{|l|}{ Sex } \\
\hline Male & $78(54.2)$ \\
\hline Female & $66(45.8)$ \\
\hline \multicolumn{2}{|l|}{ Tumor site } \\
\hline Ascending colon & $45(31.2)$ \\
\hline Transverse colon & $9(6.2)$ \\
\hline Descending colon & $12(8.4)$ \\
\hline Sigmoid & $48(33.4)$ \\
\hline Rectum & $30(20.8)$ \\
\hline \multicolumn{2}{|l|}{ Grade } \\
\hline I & $24(16.7)$ \\
\hline II & $93(64.6)$ \\
\hline III & $27(18.7)$ \\
\hline \multicolumn{2}{|l|}{ Metastasis location } \\
\hline Liver & $105(72.9)$ \\
\hline Nonregional lymph node & $15(10.4)$ \\
\hline Lung & $15(10.4)$ \\
\hline Other & $9(6.3)$ \\
\hline \multicolumn{2}{|l|}{ Familial history } \\
\hline Yes & $15(10.4)$ \\
\hline No & $129(89.6)$ \\
\hline \multicolumn{2}{|l|}{ KRAS status } \\
\hline Mutation & $75(52.1)$ \\
\hline Wild type & $69(47.9)$ \\
\hline \multicolumn{2}{|l|}{ NRAS status } \\
\hline Mutation & $6(4.2)$ \\
\hline Wild type & $138(95.8)$ \\
\hline \multicolumn{2}{|l|}{ One-year survival } \\
\hline Alive & $93(64.6)$ \\
\hline Deceased & $51(35.4)$ \\
\hline
\end{tabular}

SD - Standard deviation; KRAS - Kirsten ras;

NRAS - Neuroblastoma ras

Table 2 compares the characteristics of the patients based on KRAS status. There was just a significant difference between KRAS mutation patients and KRAS wild-type patients $(P<0.001)$ that KRAS mutation was more in the patients with age $\geq 50$ but KRAS wild type in the patients with age $<50$ years.

The comparison of 1-year overall survival based on KRAS status has been shown in Figure 1. The 6-month overall survival rate in KRAS mutation patients (84\%) was more than KRAS wild-type patients $(98.6 \%)(P=0.002)$. Furthermore, the 1-year overall survival rate was $68 \%$ in KRAS mutation patients versus $60.9 \%$ in KRAS wild-type patients $(P=0.371)$.

\section{Discussion}

This study showed that the prevalence of KRAS and NRAS 


\begin{tabular}{|c|c|c|c|}
\hline \multicolumn{4}{|c|}{$\begin{array}{l}\text { Table 2: The comparison of characteristics of the } \\
\text { patients based on KRAS status }\end{array}$} \\
\hline Variables & $\begin{array}{l}\text { KRAS mutation } \\
\qquad(n=75)(\%)\end{array}$ & $\begin{array}{c}\text { KRAS wild type } \\
(n=69)(\%)\end{array}$ & $P$ \\
\hline \multicolumn{4}{|l|}{ Age (years) } \\
\hline Mean \pm SD & $54.64 \pm 13.11$ & $51.09 \pm 12.04$ & 0.093 \\
\hline$<50 / \geq 50$ & $18(24) / 57(76)$ & $39(56.5) / 30(43.5)$ & $<0.001$ \\
\hline Sex & & & 0.645 \\
\hline Male & $42(56)$ & $36(52.1)$ & \\
\hline Female & $33(44)$ & $33(47.9)$ & \\
\hline Tumor site & & & 0.574 \\
\hline Ascending colon & $24(32)$ & $21(30.4)$ & \\
\hline Transverse colon & $6(8)$ & $3(4.3)$ & \\
\hline Descending colon & $6(8)$ & $6(8.7)$ & \\
\hline Sigmoid & $21(28)$ & $27(39.2)$ & \\
\hline Rectum & $18(24)$ & $12(17.4)$ & \\
\hline Grade & & & 0.914 \\
\hline I & $12(16)$ & $12(17.4)$ & \\
\hline II & $48(64)$ & $45(65.2)$ & \\
\hline III & $15(20)$ & $12(17.4)$ & \\
\hline Metastasis location & & & 0.436 \\
\hline Liver & $57(76)$ & $48(69.5)$ & \\
\hline $\begin{array}{l}\text { Nonregional } \\
\text { lymph node }\end{array}$ & $6(8)$ & $9(13.1)$ & \\
\hline Lung & $9(12)$ & $6(8.7)$ & \\
\hline Other & $3(4)$ & $6(8.7)$ & \\
\hline Familial history & & & 0.322 \\
\hline Yes & $6(8)$ & $9(13.1)$ & \\
\hline No & $69(92)$ & $60(86.9)$ & \\
\hline
\end{tabular}

mutations in $\mathrm{mCRC}$ patients in an Iranian population was $52.1 \%$ and $4.2 \%$, respectively. Furthermore, KRAS mutation had significantly more prevalence in lower ages compared with KRAS wild type.

One study ${ }^{[10]}$ in Western Iran on 83 mCRC patients (mean age: 57.7 years, range: $18-80$ years, and $61.4 \%$ males) and another study on $33 \mathrm{mCRC}$ patients ${ }^{[11]}$ (mean age: 51.5 years, range: $22-76$, and $79 \%$ males) in this area reported that the prevalence of KRAS mutation was $33.3 \%$ and $44.6 \%$, respectively. Furthermore, there were no significant differences between patients with KRAS mutation and wild type in $5-(69 \% \text { vs. } 64 \%)^{[10]}$ and 2-year (63\% vs. $73 \%)$ overall survivals. ${ }^{[1]}$ Two studies ${ }^{[10,11]}$ showed that the prevalence of clinicopathological factors in patients with KRAS mutation and wild type was similar. Two studies in Tehran (Central Iran) ${ }^{[1,12]}$ evaluated mCRC patients for KRAS status that the first study selected 1000 cases $(57.3 \%$ males $)$ with the prevalence of $33.6 \%$ for KRAS mutation, and the second study selected $182 \mathrm{CRC}$ patients with the prevalence of $37.4 \%$ for KRAS mutation. Koochak et al. ${ }^{[13]}$ indicate that KRAS mutations occurred at a statistically higher frequency in older patients $(>50)$ than in younger patients $(\leq 50)(P=0.0001)$. It is also worth mentioning that KRAS mutation tended to occur

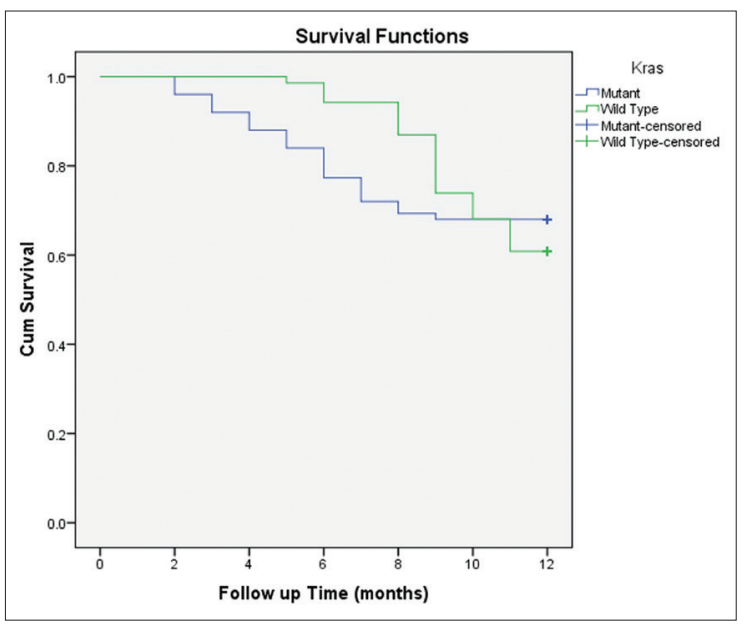

Figure 1: The comparison of 1-year overall survival based on KRAS status

at a more frequency in male cases than in female cases. KRAS mutations occur at a higher rate in pT3 than others $(P=0.0001)$. The studies in other areas of Iran reported 32\% KRAS mutation in 100 cases (mean age: 59 years and $55 \%$ males) in Southern Iran, ${ }^{[14]} 32.2 \%$ in 211 cases in Northern Iran, ${ }^{[13]}$ and $28 \%$ in 50 cases (mean age: 60.8 years), and NRAS mutation was just in one case in codon $146(2 \%)$ in Southern Iran. ${ }^{[15]}$ The prevalence of NRAS mutation in Iranian CRC patients has been reported from $0 \%{ }^{[6,10,11]}$ to $4.2 \%$ (the present study). One review study ${ }^{[7]}$ showed that the prevalence of KRAS mutation in Iran was between $30 \%$ and 50\% (few studies reported $12.5 \%-37.4 \%$ ) that this prevalence was similar to studies from other countries $(20 \%-50 \%)$.

A total of 83 mCRC patients evaluated in Saudi Arabia ${ }^{[16]}$ that $42.2 \%$ had KRAS mutation, and $51 \%$ and $23 \%$ of the tumors were from the left hemi-colon and rectum, respectively, $83 \%$ were moderately differentiated, and $86 \%$ were invasive adenocarcinoma. Another study on 300 CRC patients from Saudi Arabia ${ }^{[17]}$ reported $42 \%$ KRAS mutation that mutations were associated with advanced stage of CRC and shorter recurrence-free survival and overall survival.

The studies reported in Turkey ${ }^{[18-20]}$ checked 50, 172, and 53 mCRC patients. The prevalence of KRAS mutation was $30 \%, 44 \%$, and $49.05 \%$, respectively. KRAS mutation frequency was significantly higher in tumors located in the ascending colon, ${ }^{[18]}$ and there was no difference in progression-free survival and overall survival between KRAS mutation and KRAS wild-type patients.

One hundred and fifty CRC patients (64\% male) in Pakistan were assessed for KRAS status that $13 \%$ had the mutation, and this mutation seemed to be significantly associated with female patients. ${ }^{[21]}$ One research in Egypt $^{[22]}$ analyzed KRAS status on $26 \mathrm{mCRC}$ patients with immunohistochemistry. The results showed that $42.3 \%$ patients were KRAS mutation, and no significant 
correlation was found between clinicopathological parameters and KRAS staining results. ${ }^{[22]}$ In Oman, ${ }^{[23]} 79$ CRC patients were checked that $48.1 \%$ had mutation and no relation was noticed with wild-type or mutant KRAS with recurrence-free survival and overall survival. In Iraq, ${ }^{[2]}$ fifty CRC patients (mean age: 55.4 years and 54\% males) enrolled that $48 \%$ had KRAS mutation, and there were no significant associations of age, gender, tumor location or histology, grading, staging, or lymphovascular invasion with KRAS mutation status. In the present study, there was no significant difference between clinicopathological factors with KRAS mutation status, except for age that KRAS mutation frequency was higher in age $\geq 50$ years. Furthermore, there was no significant difference between KRAS mutation status for 1-year overall survival, but 6-month overall survival was significantly shorter in KRAS mutation compared with KRAS wild type.

\section{Conclusions}

The mCRC was more prevalent in men than women, and the mean age varied around 50-60 years. The results showed that the present study had the highest prevalence of KRAS mutation in the Middle East and Pakistan with the lowest prevalence in CRC patients. The studies reported that survival of the patients was similar between KRAS mutation and wild type, but in the present study, 6-month survival had a significant difference. In the future, studies can be considered to the survivals of shorter than 1 year in mCRC patients. In addition to, in more studies, there was no significant difference between KRAS mutation status with clinicopathological factors but can be considered to the role of age, stage, and tumor location in the future studies.

\section{Financial support and sponsorship}

Nil.

\section{Conflicts of interest}

There are no conflicts of interest.

\section{References}

1. Shahriari-Ahmadi A, Fahimi A, Payandeh M, Sadeghi M. Prevalence of oxaliplatin-induced chronic neuropathy and influencing factors in patients with colorectal cancer in Iran. Asian Pac J Cancer Prev 2015;16:7603-6.

2. Dolatkhah R, Somi MH, Bonyadi MJ, Asvadi Kermani I, Farassati F, Dastgiri S, et al. Colorectal cancer in Iran: Molecular epidemiology and screening strategies. J Cancer Epidemiol 2015;2015:643020.

3. Vatandoust S, Price TJ, Karapetis CS. Colorectal cancer: Metastases to a single organ. World J Gastroenterol 2015;21:11767-76.

4. Payandeh M, Sadeghi M, Sadeghi E. The report of KRAS mutation and NRAS wild type in a patient with thyroid metastasis from colon cancer: A Rare case report. Iran J Pathol 2016;11:71-5.

5. Van Cutsem E, Oliveira J, ESMO Guidelines Working Group.
Advanced colorectal cancer: ESMO clinical recommendations for diagnosis, treatment and follow-up. Ann Oncol 2009;20 Suppl 4:61-3.

6. Bajpai J, Susan D, Patil V, Nair R, Ghosh J, Badwe RA, et al. Taxane combination chemotherapy in breast cancer: Experience from a tertiary cancer centre in India. Indian J Med Paediatr Oncol 2017;38:18-21.

7. Payandeh M, Sadeghi M, Sadeghi E, Gholami F. Analysis of KRAS, BRAF and NRAS in patients with colorectal cancer: The first report of Western Iran. Am J Cancer Prev 2015;3:19-22.

8. Geramizadeh B. Molecular biomarkers of colorectal cancer: A review of published articles from Iran. Ann Colorectal Res 2015;3:e30100.

9. Irahara N, Baba Y, Nosho K, Shima K, Yan L, Dias-Santagata D, et al. NRAS mutations are rare in colorectal cancer. Diagn Mol Pathol 2010;19:157-63.

10. Payandeh M, Shazad B, Sadeghi M, Shahbazi M. Correlation between RAS test results and prognosis of metastatic colorectal cancer patients: A Report from Western Iran. Asian Pac J Cancer Prev 2016;17:1729-32.

11. Amirifard N, Sadeghi E, Farshchian N, Haghparast A, Choubsaz M. Evaluation of KRAS gene mutations in metastatic colorectal cancer patients in Kermanshah province. Asian Pac J Cancer Prev 2016;17:3085-8.

12. Bishehsari F, Mahdavinia $M$, Malekzadeh $R$, Verginelli $F$, Catalano $\mathrm{T}$, Sotoudeh $\mathrm{M}$, et al. Patterns of K-ras mutation in colorectal carcinomas from Iran and Italy (a Gruppo Oncologico Dell'italia Meridionale study): Influence of microsatellite instability status and country of origin. Ann Oncol 2006;17 Suppl 7:vii91-6.

13. Koochak A, Rakhshani N, Karbalaie Niya MH, Tameshkel FS, Sohrabi MR, Babaee MR, et al. Mutation analysis of KRAS and BRAF genes in metastatic colorectal cancer: A First large scale study from Iran. Asian Pac J Cancer Prev 2016;17:603-8.

14. Omidifar N Md, Geramizadeh B Md, Mirzai M Ms. K-ras mutation in colorectal cancer, A report from Southern Iran. Iran J Med Sci 2015;40:454-60.

15. Mohsen N, Ahmadreza S, Fatemeh H, Fatemeh H, Fariba ER. Frequency of K-RAS and N-RAS gene mutations in colorectal cancers in Southeastern Iran Asian Pac J Cancer Prev 2016;17:4511-5.

16. Bader T, Ismail A. Higher prevalence of KRAS mutations in colorectal cancer in Saudi Arabia: Propensity for lung metastasis. Alexandria J Med 2014;50:203-9.

17. Zekri J, Al-Shehri A, Mahrous M, Al-Rehaily S, Darwish T, Bassi S, et al. Mutations in codons 12 and 13 of K-ras exon 2 in colorectal tumors of Saudi Arabian patients: Frequency, clincopathological associations, and clinical outcomes. Genet Mol Res 2017;16:1-11.

18. Gorukmez O, Yakut T, Gorukmez O, Sag SO, Karkucak M, Kanat $\mathrm{O}$, et al. Distribution of KRAS and BRAF mutations in metastatic colorectal cancers in Turkish patients. Asian Pac J Cancer Prev 2016;17:1175-9.

19. Selcukbiricik F, Erdamar S, Ozkurt CU, Molinas Mandel N, Demirelli F, Ozguroglu M, et al. The role of K-RAS and B-RAF mutations as biomarkers in metastatic colorectal cancer. J BUON 2013;18:116-23.

20. Ozen F, Ozdemir S, Zemheri E, Hacimuto G, Silan F, Ozdemir O, et al. The proto-oncogene KRAS and BRAF profiles and some clinical characteristics in colorectal cancer in the Turkish population. Genet Test Mol Biomarkers 2013;17:135-9.

21. Murtaza BN, Bibi A, Rashid MU, Khan YI, Chaudri MS, Shakoori AR, et al. Spectrum of K ras mutations in Pakistani 
colorectal cancer patients. Braz J Med Biol Res 2014;47:35-41.

22. Elsabah MT, Adel I. Immunohistochemical assay for detection of K-ras protein expression in metastatic colorectal cancer. J Egypt Natl Canc Inst 2013;25:51-6.

23. Kumar S, Burney IA, Zahid KF, D Souza PC, Belushi MA, Mufti TD, et al. Colorectal cancer patient characteristics, treatment and survival in Oman - A single center study. Asian Pac J Cancer Prev 2015;16:4853-8.

24. Al-Allawi NA, Ismaeel AT, Ahmed NY, Merza NS. The frequency and spectrum of K-ras mutations among Iraqi patients with sporadic colorectal carcinoma. Indian J Cancer 2012;49:163-8. 